\title{
ACHADOS TIMPANOMÉTRICOS EM UM GRUPO DE ESCOLARES
}

\section{Tympanometric findings in a group of students}

\author{
Tâmara de Andrade Lindau (1), Camila Ribas Delecrode (2), Ana Claúdia Vieira Cardoso (3)
}

\begin{abstract}
RESUMO
Objetivo: caracterizar e comparar os achados timpanométricos de um grupo de escolares. Método: foram avaliados 112 escolares, de ambos os gêneros, na faixa etária de quatro anos a cinco anos e onze meses que frequentavam uma Escola Municipal de Educação Infantil, localizada em um bairro periférico da cidade de Marília. Como procedimento de triagem, utilizou-se a timpanometria. Considerou-se que a criança PASSOU na triagem quando apresentou curva timpanométrica do tipo A, bilateralmente, sendo reavaliada em caso de falha. Resultados: observou-se um alto índice de falha $(63,4 \%)$ na população estudada. Os escolares de maior faixa etária e do gênero feminino apresentaram um índice maior de timpanogramas do tipo A, em ambas as orelhas. Houve uma maior incidência de falha nas crianças de faixa etária entre 4 anos e 4 anos e 11 meses, e do gênero masculino. Conclusão: nesta amostra obteve-se um alto índice de alterações timpanométricas. Houve uma tendência de escolares do gênero masculino e de menor faixa etária apresentarem um índice maior de alterações timpanométricas quando comparados ao gênero feminino e de maior faixa etária, não sendo esta diferença estatisticamente significante.
\end{abstract}

DESCRITORES: Audição; Pré-escolar; Triagem; Orelha Média; Testes de Impedância Acústica

\section{INTRODUÇÃO}

A audição constitui-se em um pré-requisito para a aquisição e o desenvolvimento da linguagem". Qualquer privação sensorial auditiva poderá acarretar problemas nos processos comunicativos, interferindo no desenvolvimento cognitivo global, no aprendizado e nas relações interpessoais, podendo, ainda, prejudicar o desenvolvimento escolar e, consequentemente, a atuação profissional da população acometida ${ }^{1-3}$.

As otites de repetição são uma das etiologias mais frequentes de perda auditiva ${ }^{3,4}$ em crianças em fase de escolarização ${ }^{2}$. Na maioria das vezes, em torno de $70 \%$ dos casos, seria uma complicação de infecção de via aérea superior ${ }^{4-6}$.

(1) Faculdade de Filosofia e Ciências (FFC) da Universidade Estadual Paulista - UNESP, Marília, SP, Brasil.

(2) Centro de Estudos da Educação e da Saúde da Faculdade de Filosofia e Ciências (FFC) da Universidade Estadual Paulista - UNESP, Marília, SP, Brasil.

(3) Departamento de Fonoaudiologia da Faculdade de Filosofia e Ciências (FFC) da Universidade Estadual Paulista UNESP, Marília, SP, Brasil.

Conflito de interesses: inexistente
Alguns estudos referem que, além da infecção de via aérea superior, há outros fatores de risco relacionados à otite média, tais como: o cuidado em creches, sazonalidade, presença de irmãos (tamanho da família), exposição passiva ao fumo, amamentação, nível socioeconômico, atenção à saúde e uso de chupeta ${ }^{7}$. Esses fatores não estão diretamente relacionados à fisiopatologia da doença, mas, quando presentes, representam risco aumentado da doença, provavelmente por influenciar um ou mais mecanismos causais ${ }^{3,8}$.

Existem alguns comportamentos que são sugestivos da privação auditiva causados pela otite média, sendo eles: virar a cabeça em direção à fonte sonora, pedidos frequentes de repetição, intensidade vocal elevada ou reduzida, concentrar-se nos lábios da professora, apresentar desatenção, preferir o isolamento social, ter dificuldade no aprendizado, entre outros ${ }^{2}$. Tais manifestações podem passar despercebidas por pais e educadores, interferindo no desenvolvimento da linguagem e no rendimento escolar ${ }^{2,9}$.

Considerando os fatores de risco relacionados às alterações de orelha média e o impacto causado pela privação, torna-se fundamental a realização de triagem visando à detecção precoce de tais 
alterações, a fim de minimizar os prejuízos. A constatação de tais alterações foi apontada por estudo realizado com 287 escolares de cinco a dez anos de idade encontrou uma prevalência de $39,4 \%$ de falha na triagem imitanciométrica, sendo que os escolares na faixa etária de nove a dez anos falharam menos do que os mais novos e esta diferença foi estatisticamente significante ${ }^{9}$.

No que diz respeito a triagem de desordens de orelha externa e média em crianças, a American Speech and Hearing Association (ASHA) sugere os seguintes procedimentos para: obtenção de histórico clínico por meio dos pais ou responsáveis pela criança; inspeção otoscópica para visualização de anomalias estruturais óbvias, obstrução do meato acústico externo e presença de tubos de timpanostomia; timpanometria de baixa frequência (220 ou $226 \mathrm{~Hz}$ ). Nos protocolos contemporâneos, a triagem audiométrica não é empregada para identificar desordens de orelha externa e média ${ }^{10}$.

A ASHA e a American Academy of Audiology (AAA) recomendam ainda, encaminhamento médico imediato se qualquer das seguintes condições for identificada: otalgia; otorréia, patologia de orelha externa e timpanogramas planos com volume de orelha externa maior que $1.0 \mathrm{~cm}^{3}$ (sugerindo perfuração de membrana timpânica), a menos que haja tubos de ventilação ${ }^{10,11}$.

Neste sentido, a triagem auditiva é uma forma eficiente de determinar a conduta adequada a ser adotada com os escolares no que diz respeito à perda auditiva periférica e/ou às habilidades auditivas. Quando necessário, deve-se adotar condutas terapêuticas médicas e fonoaudiológicas associadas à triagem auditiva, minimizando, assim, as decorrentes consequências, principalmente no processo de aprendizagem da leitura e escrita.

Assim sendo, o presente estudo teve como objetivo caracterizar, comparar e relacionar os achados timpanométricos de um grupo de escolares.

\section{MÉTODO}

Este estudo foi realizado em uma Escola Municipal de Educação Infantil (EMEI) localizada em um bairro periférico da cidade de Marília, cuja população é de baixo nível socioeconômico. Nessa escola, é desenvolvido um Projeto de Extensão com o intuito de realizar triagem auditiva, imitanciométrica e do processamento auditivo em escolares. Neste estudo, em especial, o enfoque será os achados obtidos na triagem timpanométrica.

Foram avaliados 112 pré-escolares, de ambos os gêneros, na faixa etária de quatro anos a cinco anos e onze meses. Os pré-escolares foram distribuídos segundo gênero e faixa etária conforme a Tabela 1.

Como critérios de inclusão, foram considerados: assinatura do termo de consentimento livre e esclarecido pelos pais ou responsáveis, autorizando a participação neste estudo e a permanência da criança na EMEI, em período integral. Vale ressaltar

Tabela 1 - Distribuição dos pré-escolares segundo a variável faixa etária e gênero

\begin{tabular}{|c|c|c|c|}
\hline \multirow{2}{*}{ FAIXA ETÁRIA } & \multicolumn{2}{|c|}{ GÊNERO } & \multirow{2}{*}{ TOTAL } \\
\hline & Feminino & Masculino & \\
\hline \multirow{2}{*}{$4 a$ a $4 a 11 m$} & 29 & 32 & 61 \\
\hline & $25,90 \%$ & $28,60 \%$ & $54,50 \%$ \\
\hline \multirow{2}{*}{$5 a$ a $5 a 11 m$} & 23 & 28 & 51 \\
\hline & $20,50 \%$ & $25,00 \%$ & $45,50 \%$ \\
\hline \multirow{2}{*}{ Total } & 52 & 60 & 112 \\
\hline & $46,40 \%$ & $53,60 \%$ & $100,00 \%$ \\
\hline
\end{tabular}

que na amostra não havia crianças com sinais ou sintomas aparentes que sugerissem a presença de síndrome ou malformações craniofaciais.

Inicialmente, realizou-se a meatoscopia, a fim de identificar alterações que impedissem a realização da timpanometria e comprometessem a fidedignidade dos resultados obtidos. As alterações observadas durante a inspeção foram consideradas como critério de exclusão, sendo os pais orientados a procurar a Unidade Básica de Saúde para agendamento de consulta médica.

A imitanciometria foi realizada por meio de um analisador de orelha média Grasson - Standler GSI - 38, aferido com tom puro de corda de $226 \mathrm{~Hz}$, fone intra-auricular e sistema de sonda. A vedação do meato acústico externo foi efetuada por meio de 
uma sonda auricular acoplada a uma oliva introduzida e adaptada ao meato.

Para a análise dos achados timpanométricos, utilizou-se a classificação das curvas timpanométricas proposta por Jerger (1970) ${ }^{12}$. Considerou-se o critério PASSA/ FALHA, no qual a presença da curva timpanométrica do tipo $A$, bilateralmente, indicou que o escolar passou, e a ausência desta indicou falha nesta triagem. Nos casos de falha, o escolar foi reavaliado após seis semanas ${ }^{11}$.

Este projeto de pesquisa foi aprovado pelo Comitê de Ética em Pesquisa da Faculdade de Filosofia e Ciências - UNESP Campus de Marília, sob o protocolo $\mathrm{n}^{\mathrm{0}}$ 132/2010.

Para a descrição dos resultados, foi utilizada a análise descritiva e inferencial. $\mathrm{Na}$ análise estatística inferencial, aplicou-se o Teste da Razão de Verossimilhança, com o intuito de verificar possíveis diferenças entre os gêneros e as faixas etárias para as variáveis de interesse (tipo de curva timpanométrica e a classificação dos resultados da triagem em passa e falha). Ao analisar os resultados da associação entre as variáveis gênero e faixa etária com os resultados da triagem para o critério passa/falha percebeu-se uma tendência e, por isso, aplicou-se o Teste da Razão de Verossimilhança, ajustado pela Correção de Bonferroni, com o intuito de identificar quais combinações entre gênero e faixa etária tendiam à diferença. Adotou-se o nível de significância de $5 \%(0,005)$ para a aplicação dos testes estatísticos e a análise foi realizada utilizando o programa SPSS (Statistical Package for Social Sciences), em sua versão 19.0.

\section{RESULTADOS}

$\mathrm{Na}$ análise dos resultados, encontrou-se um alto índice de falha $(63,4 \%)$ na população estudada (Tabela 2 e 3).

Tabela 2 - Distribuição das curvas timpanométricas segundo a variável faixa etária nas orelhas direita e esquerda

\begin{tabular}{ccccccccc}
\hline \multirow{2}{*}{ Idade } & \multicolumn{4}{c}{ ORELHA DIREITA } & \multicolumn{4}{c}{ ORELHA ESQUERDA } \\
\cline { 2 - 9 } & $\mathbf{A}$ & $\mathbf{A d}$ & $\mathbf{B}$ & $\mathbf{C}$ & $\mathbf{A}$ & $\mathbf{A d}$ & $\mathbf{B}$ & $\mathbf{C}$ \\
\hline 4a a $4 \mathrm{a} 11 \mathrm{~m}$ & 23 & 0 & 22 & 16 & 23 & 0 & 26 & 12 \\
$\mathrm{~N}=61$ & $37,70 \%$ & $0,00 \%$ & $36,10 \%$ & $26,20 \%$ & $37,70 \%$ & $0,00 \%$ & $42,60 \%$ & $19,70 \%$ \\
\hline 5a a 5 a $11 \mathrm{~m}$ & 30 & 2 & 11 & 8 & 26 & 2 & 14 & 9 \\
$\mathrm{~N}=51$ & $58,80 \%$ & $3,90 \%$ & $21,60 \%$ & $15,70 \%$ & $51,00 \%$ & $3,90 \%$ & $27,50 \%$ & $17,60 \%$ \\
\hline Total & 53 & 2 & 33 & 24 & 49 & 2 & 40 & 21 \\
$\mathrm{~N}=112$ & $47,30 \%$ & $1,80 \%$ & $29,50 \%$ & $21,40 \%$ & $43,80 \%$ & $1,80 \%$ & $35,70 \%$ & $18,80 \%$ \\
\hline \multicolumn{8}{c}{$\mathbf{p = 0 , 0 2 6 ^ { * }}$} \\
\hline
\end{tabular}

Teste da Razão de Verossimilhança / p-valor $<0,005$ = estatisticamente significante*

Tabela 3 - Distribuição das curvas timpanométricas segundo a variável gênero nas orelhas direita e esquerda

\begin{tabular}{ccccccccc}
\hline \multirow{2}{*}{ Gênero } & \multicolumn{4}{c}{ ORELHA DIREITA } & \multicolumn{5}{c}{ ORELHA ESQUERDA } \\
\cline { 2 - 9 } & $\mathbf{A}$ & Ad & B & C & A & Ad & B & C \\
\hline Feminino & 33 & 0 & 13 & 6 & 25 & 0 & 16 & 11 \\
$\mathrm{~N}=52$ & $63,50 \%$ & $0,00 \%$ & $25,00 \%$ & $11,50 \%$ & $48,10 \%$ & $0,00 \%$ & $30,80 \%$ & $21,20 \%$ \\
\hline Masculino & 20 & 2 & 20 & 18 & 24 & 2 & 24 & 10 \\
$\mathrm{~N}=60$ & $33,30 \%$ & $3,30 \%$ & $33,30 \%$ & $30,00 \%$ & $40,00 \%$ & $3,30 \%$ & $40,00 \%$ & $16,70 \%$ \\
\hline Total & 53 & 2 & 33 & 24 & 49 & 2 & 40 & 21 \\
$\mathrm{~N}=112$ & $47,30 \%$ & $1,80 \%$ & $29,50 \%$ & $21,40 \%$ & $43,80 \%$ & $1,80 \%$ & $35,70 \%$ & $18,80 \%$ \\
\hline \multicolumn{1}{c}{$\mathbf{p = 0 , 0 0 4}$} \\
\hline
\end{tabular}

Teste da Razão de Verossimilhança / p-valor $<0,005$ = estatisticamente significante ${ }^{\star}$ 
Ao considerar as variáveis faixa etária (Tabela 2) e gênero (Tabela 3), observou-se que os escolares de menor faixa etária e do gênero masculino apresentaram um índice menor de timpanogramas do tipo A, em ambas as orelhas, quando comparados aos escolares de maior faixa etária e do gênero feminino, sendo esta diferença estatisticamente significante apenas na orelha direita para ambas as variáveis.
Quanto ao critério passa/falha nas duas faixas etárias (Tabela 4) e em ambos os gêneros (Tabela 5), os resultados demonstraram uma maior incidência de falha na faixa etária de quatro anos a quatro anos e onze meses, e no gênero masculino, porém, esta diferença não foi estatisticamente significante para nenhuma variável.

Tabela 4 - Ocorrência de passa/falha na timpanometria segundo a variável faixa etária

\begin{tabular}{cccc}
\hline \multirow{2}{*}{ Idade } & \multicolumn{2}{c}{ TIMPANOMETRIA } & \multirow{2}{*}{ Total } \\
\cline { 2 - 4 } & Passa & Falha & 61 \\
\multirow{2}{*}{ 4a a 4a11m } & 18 & 43 & $100,00 \%$ \\
\hline \multirow{2}{*}{ 5a a 5a11m } & $29,50 \%$ & $70,50 \%$ & 51 \\
& 23 & 28 & $100,00 \%$ \\
\hline \multirow{2}{*}{ Total } & $45,10 \%$ & $54,90 \%$ & 112 \\
& 41 & 71 & $100,00 \%$ \\
\hline
\end{tabular}

Teste da Razão de Verossimilhança / p-valor $<0,005$ = estatisticamente significante*

Tabela 5 - Ocorrência de passa/falha na timpanometria segundo a variável gênero

\begin{tabular}{cccc}
\hline \multirow{2}{*}{ Gênero } & \multicolumn{2}{c}{ TIMPANOMETRIA } & \multirow{2}{*}{ Total } \\
\cline { 2 - 4 } & Passa & Falha & 52 \\
\multirow{2}{*}{ Feminino } & 24 & 28 & $100,00 \%$ \\
\hline \multirow{2}{*}{ Masculino } & $46,20 \%$ & $53,80 \%$ & 60 \\
& 17 & 43 & $100,00 \%$ \\
\hline \multirow{2}{*}{ Total } & $28,30 \%$ & $71,70 \%$ & 112 \\
& 41 & 71 & $100,00 \%$ \\
\hline
\end{tabular}

Teste da Razão de Verossimilhança / p-valor $<0,005$ = estatisticamente significante ${ }^{\star}$

Ao combinar as variáveis gênero e faixa etária (Figura 1) segundo o critério passa/falha, verificou-se que escolares de ambos os gêneros, entre quatro anos e quatro anos e onze meses, e do gênero masculino, entre cinco anos e cinco anos e onze meses, falharam mais do que os escolares do gênero feminino nesta faixa etária.

Essa tendência foi estatisticamente significante apenas na comparação de escolares do gênero feminino na maior faixa etária com escolares do gênero masculino na menor faixa etária (Tabela 6). Logo, pode-se afirmar que existe uma tendência de que mais escolares do gênero feminino, de faixa etária entre cinco anos e cinco anos e onze meses, não apresentem alteração de orelha média em relação aos escolares do gênero masculino de faixa etária entre quatro anos a quatro anos e onze meses. 


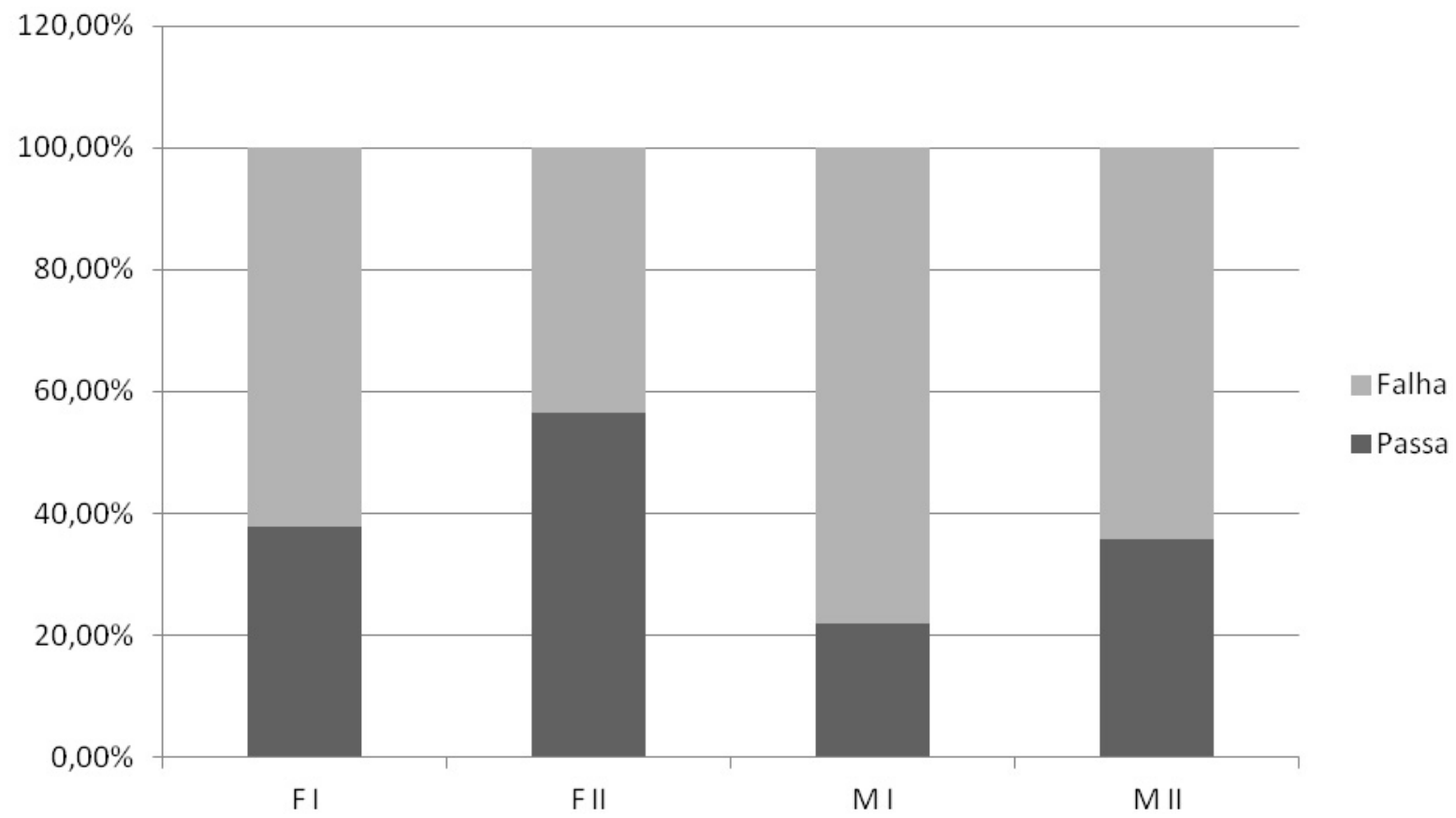

Legenda: F- feminino/ M - masculino/ I - 4 anos a 4 anos e 11 meses / II - 5 anos a 5 anos e 11 meses.

Figura 1 - Distribuição de escolares segundo o gênero e faixa etária para o critério passa/falha na triagem timpanométrica

Tabela 6 - Análise estatística do critério passa/falha na triagem timpanométrica combinando gênero e faixa etária dos escolares

\begin{tabular}{cc}
\hline Par de Categorias & Significância $(\mathbf{p})$ \\
\hline Fem(4a a 4a11m) $\times$ Fem(5a a 5a11m) & 0,181 \\
Fem(4a a 4a11m) x Mas(4a a 4a11m) & 0,169 \\
Fem(4a a 4a11m) x Mas(5a a 5a11m) & 0,862 \\
Fem(5a a 5a11m) x Mas(4a a 4a11m) & $\mathbf{0 , 0 0 8 ^ { * }}$ \\
Fem(5a a 5a11m) x Mas(5a a 5a11m) & 0,136 \\
Mas(4a a 4a11m) x Mas(5a a 5a11m) & 0,235 \\
\hline
\end{tabular}

Legenda: Fem= feminino / Mas= masculino

Teste da Razão de Verossimilhança ajustado pela Correção de Bonferroni / alfa de Bonferroni $=0,008512-$ estatisticamente significante*

\section{DISCUSSÃO}

A triagem auditiva é uma forma eficiente de determinar a conduta adequada a ser adotada com os escolares no que diz respeito à perda auditiva periférica e/ou às habilidades auditivas. Associado à triagem auditiva, devem-se adotar condutas terapêuticas médicas e fonoaudiológicas, quando necessário, minimizando, assim, as decorrentes consequências, principalmente no processo de aprendizagem da leitura e escrita ${ }^{9}$.
Sendo assim, neste estudo os pré-escolares que permaneceram com falha no reteste da triagem timpanométrica, após orientação aos pais, foram encaminhados para avaliação médica na Unidade Básica de Saúde próxima à escola.

A detecção precoce e a intervenção imediata em crianças com privação auditiva aumentam a probabilidade de otimizar o potencial de linguagem receptiva e expressiva, de alfabetização (leitura e escrita), desempenho acadêmico, desenvolvimento emocional e social ${ }^{9}$. 
$\mathrm{Na}$ literatura foram encontrados poucos estudos referentes à triagem auditiva em escolares na última década. Com relação às alterações de orelha média, a maioria dos estudos enfocou a eficácia de antibióticos no tratamento e as possíveis causas destas alterações ${ }^{3-6,13,14}$.

Neste estudo, obteve-se um alto índice de alterações na triagem timpanométrica. Tal fato pode ser explicado pela presença de vários fatores de risco ao qual esta população está exposta, tais como: cuidados em creche ${ }^{7}$, variações climáticas ${ }^{7}$, nível socio-econômico ${ }^{3,7,8}$ e rinorréia persistente ${ }^{15}$.

Os fatores de risco não estão diretamente envolvidos na fisiopatologia da otite média, mas resultam, quando presentes, em risco aumentado da doença, provavelmente por influenciarem um ou mais mecanismos causais ${ }^{7}$.

As otites de repetição são uma das etiologias mais frequentes de perda auditiva ${ }^{3,4}$ em crianças em fase de escolarização². Na maioria das vezes, em torno de $70 \%$ dos casos, seria uma complicação de infecção de via aérea superior ${ }^{4-6}$.

Alguns estudos relataram que a otite média é uma doença comum em países em desenvolvimento $^{3-15}$, tal como ocorre no Brasil, e a variabilidade dos fatores de risco entre os países é um reflexo das diferenças socioculturais da população estudada $^{16}$.

Neste estudo a prevalência de alterações timpanométricas foi de $63,4 \%$, sendo encontrada na literatura consultada a prevalência variando de $39,4 \%^{9}$ a $74,8 \%{ }^{3}$.

Ao analisar qualitativamente as alterações timpanométricas encontrou-se um predomínio de curvas do tipo $\mathrm{B}$ e $\mathrm{C}$, sendo tais achados semelhantes ao encontrados na literatura ${ }^{9,17}$, sendo o primeiro realizado com 287 escolares de cinco a dez anos, que demonstrou curvas timpanométricas do tipo Ar, B e C em $20 \%$ das crianças avaliadas ${ }^{9}$, e o segundo que avaliou 142 crianças da mesma faixa etária, que encontrou $46 \%$ de timpanometrias alteradas, predominando curvas do tipo $\mathrm{B}, \mathrm{C}$ e As, revelando em ambos os estudos ocorrência de alterações na orelha média ${ }^{17}$.

Em relação ao gênero, observou-se uma maior incidência de timpanogramas alterados nos escolares do gênero masculino, sendo esta diferença estatisticamente significante apenas na orelha direita. Em estudos de outros autores não foi encontrada associação entre a variável gênero e a maior prevalência de otite média em um desses gêneros ${ }^{3,9,15}$.

Quanto à variável faixa etária, observou-se que escolares mais novos falharam mais do que os mais velhos, no entanto, esta diferença não foi estatisticamente significante. Na literatura consultada não há consenso quanto à idade como fator de risco para otite média, sendo, que, alguns autores associaram o fator idade a maior prevalência de otite média ${ }^{3,18}$ e outros não9, 15 .

Estudo realizado com 555 crianças afirmou que as crianças das séries iniciais são mais propensas ao diagnóstico positivo de otite do que as de séries superiores $^{3}$. Porém, um programa de triagem auditiva desenvolvido com escolares de 5 a 10 anos concluiu que a idade não foi um fator de risco significante para alterações de orelha média ${ }^{9}$.

A otite média é uma doença altamente prevalente na infância, com maior pico de incidência entre 6 e 24 meses de idade e com segundo pico de incidência entre 4 e 7 anos $^{19}$.

Os parâmetros raça, sexo e idade influenciam na estrutura da tuba auditiva ou sua função, enquanto o fator idade também determina resposta imunológica do hospedeiro, sendo evidente que alguns fatores estão relacionados, pois são crianças menores que apresentam maior número de infecções de vias aéreas superiores ${ }^{7}$.

Autores $^{20}$ afirmam que a privação sensorial decorrente da otite média serosa, agravada pelo número e pela duração dos episódios da doença, pode afetar a percepção da fala e dificultar a compreensão, principalmente em ambiente ruidoso, e até mesmo prejudicar o desenvolvimento da linguagem da criança. A flutuação da audição causada por alterações da orelha média nos primeiros anos de vida pode levar a alterações no processamento auditivo, interferindo no aprendizado escolar.

Algumas medidas poderiam ser implantadas com o objetivo de reduzir a incidência de alterações de orelha média. Autores sugerem como ações: o tratamento de adenoide aumentada, sinusites crônicas e alergias como algumas das medidas necessárias para reduzir o problema da rinorréia persistente, considerando que visitas regulares as escolas visando à triagem de crianças com rinorréia persistente, respiração oral e otite média deveriam fazer parte de um programa nacional enfatizando a saúde auditiva e orientações quanto ao efeito do fumo passivo no sistema respiratório e auditivo de crianças deveriam ser enfatizados em todas as campanhas anti-fumo ${ }^{15}$.

Considerando os achados deste estudo, faz-se necessária a implementação de ações que visem reduzir a incidência de alterações de orelha média nesta população. Desta forma, sugere-se que pais e professores sejam orientados e haja a implantação de programas de imunização desta população, visando reduzir os episódios de infecções de vias aéreas superiores. 


\section{CONCLUSÃO}

Nesta amostra, obteve-se um alto índice de alterações timpanométricas. Houve uma tendência de escolares do gênero masculino e de menor faixa etária apresentarem um índice maior de alterações timpanométricas quando comparados ao gênero feminino e de maior faixa etária, não sendo esta diferença estatisticamente significante.

\begin{abstract}
Purpose: to characterize and compare the tympanometric findings in a group of preschoolers. Method: 112 preschoolers were evaluated, both genders, aged from four years old to five years and eleven months old who attended a Municipal School of Early Childhood Education in the suburbs of Marilia city- SP. Tympanometry was used as triage procedure. It was considered that the child PASSED in the triage when it presented an A type tympanometric curve, bilaterally, being reevaluated in case of failure. Results: it was observed a high failure index (63.4\%) in the studied population. The older female preschoolers presented a higher index of $A$ type tympanograms, in both ears. There was a higher incidence of failure in male preschoolers aged from four years to four years and eleven months. Conclusion: in this sample, there was a high index of tympanometric alteration. There was a tendency among younger male students to present a higher index of tympanometric alteration when compared to older female preschoolers, this difference was not statistically significant.
\end{abstract}

KEYWORDS: Hearing; Preschool; Triage; Middle Ear; Acoustic Impedance Tests

\section{REFERÊNCIAS}

1. Gatto $\mathrm{Cl}$, Tochetto TM. Deficiência auditiva infantil: implicações e soluções. Rev CEFAC. 2007;9(1):110-5.

2. Vieira ABC, Macedo LR, Gonçalves DU. O diagnóstico da perda auditiva na infância. Rev. Pediatria. 2007;29(1):43-9.

3. Taha AA, Pratt SR, Farahat TM, Abdel-Rasoul GM, Albtanony MA, Elrashiedyet ALE et al. Prevalence and risk factors of hearing impairment among primary-school children in Shebin El-kom District, Egypt. Am J Audiol. 2010;19:46-60.

4. Spiro DM, King WD, Arnold DH, Johnston C, Baldwin S. A randomized clinical trial to assess the effects of tympanometry on the diagnosis and treatment of acute otitis media. Pediatrics. 2004;114(1):177-81.

5. Winther B, Alper CM, Mandel EM, Doyle WJ, Hendley JO. Temporal relationships between colds, upper respiratory viruses detected by polymerase chain reaction, and otitis media in young children followed through a typical cold season. Pediatrics. 2007;119(6):1069-75.

6. Revai K, Patel JA, Grady JJ, Chonmaitree $\mathrm{T}$. Tympanometric findings in young children during upper respiratory tract infections with and without acute otitis media. Pediatr Infect Dis $\mathrm{J}$. 2008;27:292-5.
7. Lubianca Neto JF, Hemb L, Silva DB. Fatores de risco para otite média aguda recorrente: onde podemos intervir?- uma revisão sistemática da literatura. J Pediatr. 2006;82(2):87-96.

8. Adhikari $P$. Otite média crônica supurada em crianças de escolas do vale katmandu. Arq. Int. Otorrinolaringol. 2007;11(2):175-8.

9. Colella-Santos MF, Bragato GR, Martins PMF, Dias AB. Triagem auditiva em escolares de 5 a 10 anos. Rev. CEFAC. 2009;11(4):644-53.

10. American Academy of Audiology (AAA) Position Statement: Identification of hearing loss and middle-ear dysfunction in preschool and school-age children. Audiology Today. 1997;9:21-3.

11. American Speech-Language-Hearing Association (ASHA). Guidelines for Audiologic Screening. Rockville Pike.1997.

12. Jerger J. Clinical experience with impedance audiometry. Arch Otorhinolaringol. 1970;92:311-24. 13. Meropol SB, Glick HA, Asch DA. Age Inconsistency in the American Academy of Pediatrics Guidelines for Acute Otitis Media. Pediatrics. 2008;121(4):657-68.

14. Vergison A, Dagan R, Arguedas A, Bonhoeffer $\mathrm{J}$, Cohen R, DHooge I et al. Otitis media and its consequences: beyond the earache. Lancet Infect Dis. 2010; 10(3):195-203

15. Sophia A, Isaac R, Rebekah G, Brahmadathan K, Rupa V. Risk factors for otitis media among 
preschool, rural Indian children. Int. J. Pediatric Otorhinolaryngol. 2010;74(6):677-83.

16. Rovers MM, Kok IMCM, Schilder AGM. Risk factors for otitis media: an international perspective. Int J Pediatr. Otorhinolaryngol. 2006;70:1251-6.

17. Guida HL, Diniz TH. Perfil audiológico em crianças de 5 a 10 anos de idade. Arq. Int. Otorrinolaringol. 2008;12(2):224-9.

18. Caylan, R, Bektas, D, Atalay, C, Korkmaz, O. Prevalence and risk factors of otits media with effusion in Trabzon, a city in nrtheastern Turkey, with an emphasis on the recommendation of OME screening. Eur. Arch. Otorhinolaryngol. 2006;263: 404-8.

19. Almeida CIR, Almeida RR. Otite media aguda. In: Campos $\mathrm{CAH}$, Costa $\mathrm{HOO}$, editores. Tratado de Otorrinolaringologia. 1르 ed. São Paulo: Roca; 2003. Vol. 2, p. 21-7.

20. Pereira PKS, Azevedo MF, Testa JR. Alterações condutivas em neonatos que falharam na triagem auditiva neonatal. Braz. J. Otorhinolaryngol. 2010;76:347-54.

Endereço para correspondência:

Tâmara de Andrade Lindau

Rua José Chieramont, 54

Santa Rosa de Viterbo - SP - Brasil

CEP: 14270-000

E-mail: lindau.fono@gmail.com 